\title{
Equivalent Rise Time for Resonance in Power/Ground Noise Estimation
}

\author{
Emre Salman, Eby G. Friedman \\ Department of Electrical and Computer Engineering \\ University of Rochester \\ Rochester, New York 14627 \\ [salman, friedman]@ece.rochester.edu
}

\author{
Radu M. Secareanu, Olin L. Hartin \\ Freescale Semiconductor \\ MMSTL \\ Tempe, Arizona 85284 \\ [r54143,lee.hartin]@freescale.com
}

\begin{abstract}
The non-monotonic behavior of power/ground noise with respect to the rise time $t_{r}$ is investigated for an inductive power distribution network with a decoupling capacitor. A time domain solution is provided for the rise time that produces resonant behavior, thereby maximizing the power/ground noise. The sensitivity of the ground noise to the decoupling capacitance $C_{d}$ and parasitic inductance $L_{g}$ is evaluated as a function of the rise time. Increasing the decoupling capacitance is shown to efficiently reduce the noise for $t_{r} \leq 2 \sqrt{L_{g} C_{d}}$. Alternatively, reducing the parasitic inductance $L_{g}$ is shown to be effective for $t_{r} \geq 2 \sqrt{L_{g} C_{d}}$.
\end{abstract}

\section{INTRODUCTION}

The distribution of robust power supply and ground voltages is a challenging task in modern integrated circuits due to scaled power supply voltages and the increased switching activity of the load circuit [1], [2]. The parasitic resistance and inductance of the power and ground distribution networks produce $I R+$ $L \partial i / \partial t$ voltage drops, reducing the overall voltage at the load.

The changing voltages increase the delay uncertainty while reducing the noise margin in high performance integrated circuits, possibly causing a timing violation or logic failure. Decoupling capacitors are often used to reduce power/ground noise by decreasing the overall impedance of the power distribution network [3], [4], [5].

The parallel combination of the decoupling capacitor $C_{d}$ with the parasitic inductance $L_{g}$ of the power distribution network, however, produces a peak impedance at the resonant frequency $f=1 /\left(2 \pi \sqrt{L_{g} C_{d}}\right)$ due to the $L C$ tank circuit. The impedance at the resonant frequency should, therefore, be smaller than the target impedance to satisfy noise constraints. The impedance characteristics of a power distribution system have been investigated with particular focus on the resonant behavior [6], [7]. The corresponding rise time that produces the maximum noise in the time domain, however, has not received much attention.

The non-monotonic behavior of power/ground noise with respect to the rise time is investigated in this paper. A worst

This research is supported in part by the Semiconductor Research Corporation under Contract No. 2004-TJ-1207, the National Science Foundation under Contract Nos. CCR-0304574 and CCF-0541206, grants from the New York State Office of Science, Technology \& Academic Research to the Center for Advanced Technology in Electronic Imaging Systems, and by grants from Intel Corporation, Eastman Kodak Company, Intrinsix Corporation, and Freescale Semiconductor Corporation.

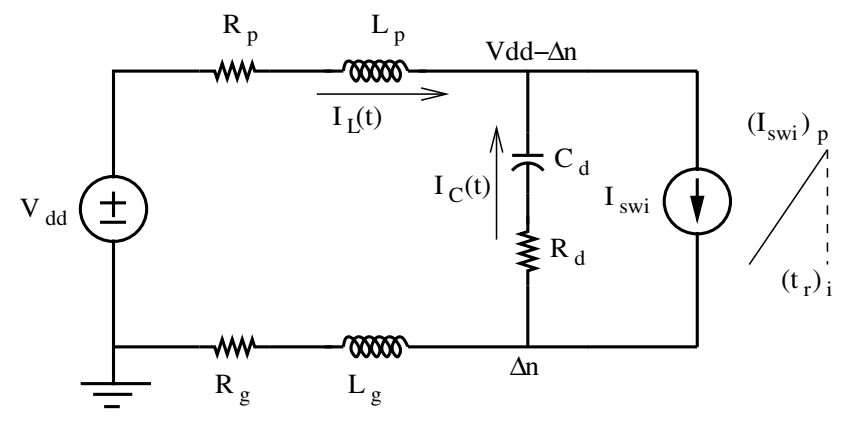

Fig. 1. Equivalent circuit model to estimate power supply noise and ground bounce. $R_{p}, L_{p}$, and $R_{g}, L_{g}$ represent the power and ground rail impedances, respectively. $C_{d}$ is the decoupling capacitor and $R_{d}$ is the effective series resistance (ESR) of the capacitor. The load circuit is represented by a current source with a rise time $\left(t_{r}\right)_{i}$ and peak current $\left(I_{s w i}\right)_{p}$.

case rise time producing the maximum noise in the presence of a decoupling capacitor is presented. The sensitivity of the noise to the decoupling capacitance and parasitic inductance is also evaluated.

The rest of the paper is organized as follows. The equivalent circuit model to estimate the peak-to-peak power/ground noise is described in Section II. The non-monotonic noise behavior with respect to the rise time is investigated in Section III. The paper is concluded in Section IV.

\section{Power/Ground NoISE Model}

The equivalent circuit model to investigate the noise behavior with respect to the rise time is shown in Fig. 1, where $R_{p}, L_{p}$, and $R_{g}, L_{g}$ represent the power and ground rail impedances, respectively. $C_{d}$ is the decoupling capacitor and $R_{d}$ is the effective series resistance (ESR) of the capacitor. The load circuit is represented by a current source with a rise time $\left(t_{r}\right)_{i}$ and peak current $\left(I_{s w i}\right)_{p}$. The current provided by the decoupling capacitance $I_{C}(t)$ and the current flowing through the parasitic inductance $I_{L}(t)$ from the power supply are, respectively,

$$
\begin{gathered}
I_{C}(t)=-C_{d} \frac{\partial V_{C}}{\partial t} \\
I_{L}(t)=\frac{1}{L_{g}} \int_{0}^{t} V_{L}(t) \partial t
\end{gathered}
$$


where $V_{C}(t)$ and $V_{L}(t)$ are, respectively,

$$
\begin{gathered}
V_{C}(t)=V_{d d}-2 \Delta n+I_{C}(t) R_{d}, \\
V_{L}(t)=\Delta n-I_{L}(t) R_{p} .
\end{gathered}
$$

Assuming a ramp function for the noise $\Delta n(t)=$ $\left[\left(V_{\text {gnd }}\right)_{p} /\left(t_{r}\right)_{v}\right] t$, replacing (3) in (1) and (4) in (2), and taking the derivative with respect to time results in the following differential equations,

$$
\begin{gathered}
I_{C}(t)=\frac{2 C_{d}\left(V_{g n d}\right)_{p}}{\left(t_{r}\right)_{v}}-R_{d} C_{d} \frac{\partial I_{C}(t)}{\partial t}, \\
\frac{\partial I_{L}(t)}{\partial t}=\frac{\left(V_{g n d}\right)_{p} t}{L_{g}\left(t_{r}\right)_{v}}-\frac{R_{p}}{L_{g}} I_{L}(t) .
\end{gathered}
$$

Solving these differential equations with the initial conditions $I_{C}(0)=0$ and $I_{L}(0)=0$ produces the inductive and capacitive current, respectively,

$$
\begin{gathered}
I_{C}(t)=\left(V_{g n d}\right)_{p} \frac{2 C_{d}}{t_{r}}\left(1-e^{-t /\left(R_{d} C_{d}\right)}\right), \\
I_{L}(t)=\left(V_{g n d}\right)_{p}\left[\frac{t}{t_{r} R_{g}}-\frac{L}{t_{r} R_{g}^{2}}\left(1-e^{-t / \frac{L}{R_{g}}}\right)\right] .
\end{gathered}
$$

These currents can be rewritten as

$$
\begin{aligned}
& I_{C}(t)=G_{C}(t)\left(V_{g n d}\right)_{p}, \\
& I_{L}(t)=G_{L}(t)\left(V_{g n d}\right)_{p},
\end{aligned}
$$

where $G_{C}(t)$, the conductance of the capacitance path, and $G_{L}(t)$, the conductance of the inductance path, are given, respectively, by

$$
\begin{gathered}
G_{C}(t)=\frac{2 C_{d}}{\left(t_{r}\right)_{v}}\left(1-e^{-t /\left(R_{d} C_{d}\right)}\right), \\
G_{L}(t)=\frac{t}{\left(t_{r}\right)_{v} R_{g}}-\frac{L_{g}}{\left(t_{r}\right)_{v} R_{g}^{2}}\left(1-e^{-t / \frac{L_{g}}{R_{g}}}\right) .
\end{gathered}
$$

Note that these conductances are both a function of the rise time $\left(t_{r}\right)_{v}$. Specifically, as the rise time becomes smaller, $G_{C}(t)$ increases and $G_{L}(t)$ decreases. The capacitive current, therefore, increases with decreasing rise time. Alternatively, the inductive current increases with longer rise times. Intuitively, a smaller rise time corresponds to a higher frequency, where the impedance of the capacitance is smaller and the inductance is higher. The capacitance is, therefore, more effective at smaller rise times and becomes less effective as the rise time increases.

Assuming the peak noise occurs when the switching current reaches the maximum current, e.g., $\left(t_{r}\right)_{v}=\left(t_{r}\right)_{i}=t_{r}$, the peak ground noise at $t=t_{r}$ can be expressed as

$$
\frac{1}{\left(V_{\text {gnd }}\right)_{p}}=\frac{G_{C}\left(t_{r}\right)}{\left(I_{s w i}\right)_{p}}+\frac{G_{L}\left(t_{r}\right)}{\left(I_{s w i}\right)_{p}} .
$$

Replacing (11) and (12) in (13) produces

$$
\left(V_{g n d}\right)_{p}=\frac{\left(I_{s w i}\right)_{p} R_{g}^{2} t_{r}}{2 C_{d} R_{g}^{2}\left(1-e^{-t_{r} /\left(R_{d} C_{d}\right)}\right)-L\left(1-e^{-t_{r} / L_{g}}\right)+R_{g} t_{r}} .
$$

Note that if the capacitive current is much greater than the inductive current, e.g., (9) $\gg(10)$ or $(11) \gg(12)$, the second term in (13) can be neglected without a significant loss in accuracy, guaranteeing the pessimism of the expression. In this case, the peak ground noise is approximated by

$$
\left(V_{g n d}\right)_{p} \approx\left(I_{s w i}\right)_{p} / G_{C}\left(t_{r}\right) .
$$

Alternatively, if the inductive current is much greater, the first term in (13) can be neglected and the peak noise is estimated as

$$
\left(V_{g n d}\right)_{p} \approx\left(I_{s w i}\right)_{p} / G_{L}\left(t_{r}\right) .
$$

If the circuit is underdamped, i.e., the damping factor is smaller than one, oscillations occur due to a parallel combination of the parasitic inductance and decoupling capacitor. In this case, the peak-to-peak ground noise voltage is

$$
\left(V_{g n d}\right)_{p p}=\left(V_{g n d}\right)_{p}\left[1+e^{-\pi \zeta / \sqrt{1-\zeta^{2}}}\right],
$$

where $\zeta=\left[\left(2 R_{g}+R_{d}\right) / 2\right] \sqrt{C_{d} / 2 L_{g}}$ is the damping factor.

\section{NON-MONOTONIC NOISE Behavior}

The impedance of a parallel $L C$ circuit is maximum at the resonant frequency, $\omega=1 / \sqrt{(L C)}$. At this frequency, both the capacitive and inductive paths carry a significant amount of current, giving rise to resonant behavior. Similarly, in the time domain, there exists a rise time at which the capacitive and inductive currents are close and the peak-to-peak noise is maximum. The worst case rise time producing the maximum noise is described in Section III-A. The sensitivity of the noise to the decoupling capacitance and parasitic inductance as a function of the rise time is investigated in Section III-B.

\section{A. Worst Case Rise Time}

The capacitive and inductive currents and the corresponding ground noise are plotted as a function of rise time in Fig. 2 using (7) and (8) for the currents, and (17) for the noise voltage when $\left(I_{s w i}\right)_{p}=11.5 \mathrm{~mA}, L_{g}=1 \mathrm{nH}, C_{d}=10 \mathrm{pF}, R_{g}=2.2 \Omega$, and $R_{d}=0.1 \Omega$. Equation (17) is also compared with SPICE in Fig. 2.

The model accurately captures the non-monotonic dependence of noise on rise time, exhibiting a maximum error of $12.5 \%$. As shown in Fig. 2, for sufficiently small rise times, the capacitive current dominates, and the inductance does not affect the ground noise. As the rise time increases, the capacitive current decreases and the inductive current increases. The peak noise occurs at a rise time where these currents are approximately equal, denoted as the worst case rise time (similar to the resonant behavior in the frequency domain). If the rise time increases further, the noise decreases due to a lower $L \partial i / \partial t$ noise, making the capacitance ineffective. The assumption of fast transients as the worst case scenario for noise can be overly optimistic in a circuit with sufficient decoupling. This conclusion is similar to reducing the resonant frequency with a larger capacitance. Increasing the decoupling capacitance, therefore, has the drawback of 


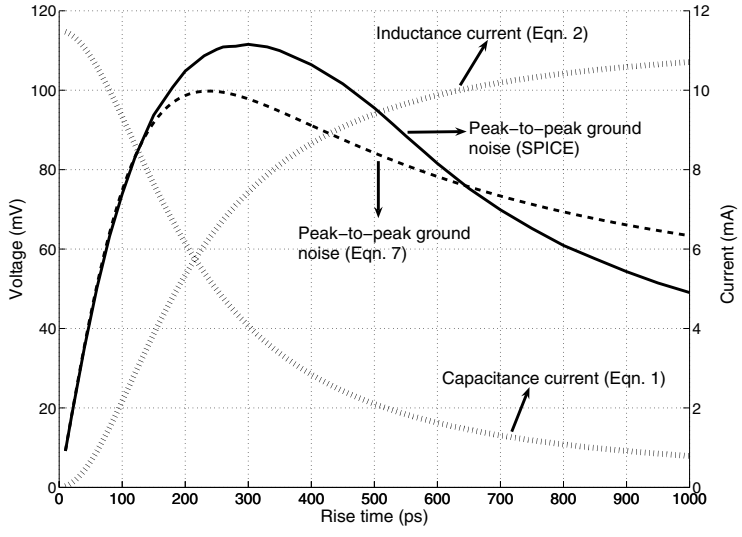

Fig. 2. Comparison of peak-to-peak ground noise as a function of the rise time obtained from SPICE simulations and (17) for $\left(I_{s w i}\right)_{p}=11.5 \mathrm{~mA}$. The ground network impedances are $L_{g}=1 \mathrm{nH}, C_{d}=10 \mathrm{pF}, R_{g}=2.2 \Omega$, and $R_{d}=0.1 \Omega$. The dotted lines depict the estimated capacitive and inductive currents as a function of the rise time.

reducing the resonant frequency, or similarly, increasing the worst case rise time.

According to Fig. 2, the maximum peak-to-peak noise occurs at the rise time where the inductive and capacitive currents are approximately equal. Thus, an expression for the worst case rise time can be developed by equating (11) with (12) at $t=t_{r}$ and solving for $t_{r}$,

$$
G_{C}\left(t_{r}\right)-G_{L}\left(t_{r}\right)=0
$$

A closed form solution, however, does not exist due to the exponential terms in $G_{C}\left(t_{r}\right)$ and $G_{L}\left(t_{r}\right)$. Assuming $R_{d} \rightarrow 0$, $G_{C}\left(t_{r}\right) \approx \frac{2 C_{d}}{t_{r}}$ since $e^{-t_{r} /\left(R_{d} C_{d}\right)} \rightarrow 0$. Similarly, assuming $R_{g} \rightarrow$ $0, G_{L}\left(t_{r}\right) \approx \frac{t_{r}}{2 L_{g}}$ from a Taylor series expansion. The rise time $t_{r}$ at which these conductances are approximately equal is, therefore,

$$
\frac{2 C_{d}}{t_{r}}=\frac{t_{r}}{2 L_{g}} \Longrightarrow t_{r}=2 \sqrt{\left(L_{g} C_{d}\right)} .
$$

The ground noise is obtained at $t_{r}=2 \sqrt{\left(L_{g} C_{d}\right)}$ for different parasitic impedances of the ground network and compared with the maximum noise obtained at the same impedance. These results are listed in Table I. The error of $t_{r}=2 \sqrt{\left(L_{g} C_{d}\right)}$ in estimating the maximum noise is greater with increasing $R_{g}$ and $R_{d}$, but is sufficiently small within the practical values of these resistances, as listed in Table I.

The effect of the parasitic resistance $R_{g}$ and the ESR of the decoupling capacitance $R_{d}$ on the worst case rise time is further illustrated, respectively, in Figs. 3 and 4. Increasing $R_{g}$ reduces the noise until a specific rise time is reached due to additional damping. Beyond this rise time, however, the noise increases due to a greater $I R$ drop on the ground network, making the decoupling capacitance ineffective. Alternatively, an increase in $R_{d}$ results in decreased noise at higher rise times due to the increased damping and higher noise at smaller rise times where the decoupling capacitance is effective.

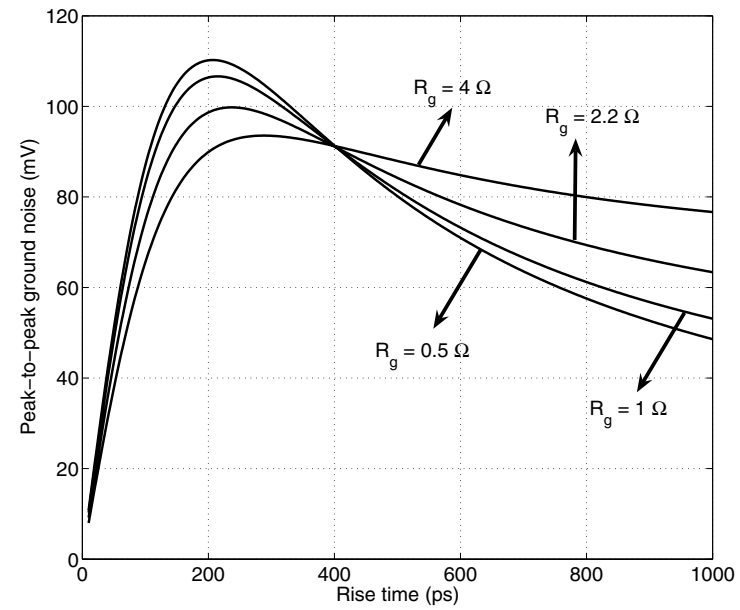

Fig. 3. Peak-to-peak ground noise for different values of $R_{g}$ when $\left(I_{s w i}\right)_{p}=$ $11.5 \mathrm{~mA}, L_{g}=1 \mathrm{nH}, \mathrm{C}_{d}=10 \mathrm{pF}$, and $R_{d}=0.1 \Omega$

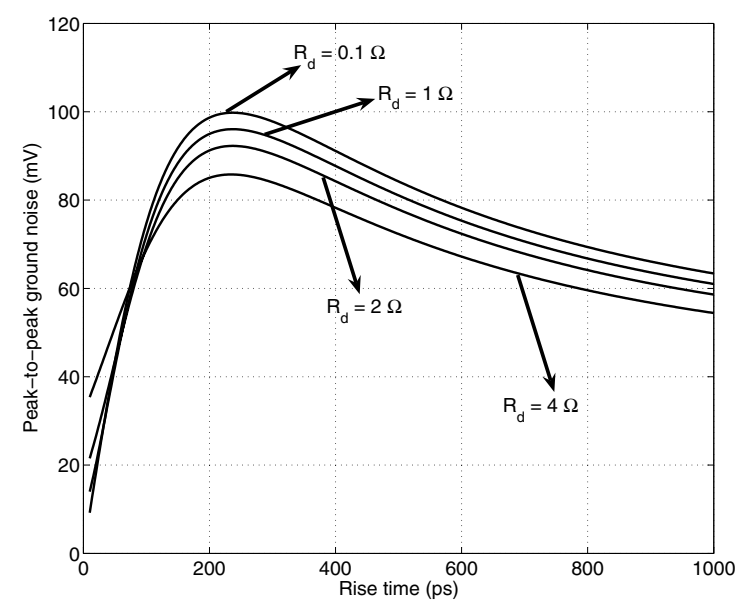

Fig. 4. Peak-to-peak ground noise for different values of $R_{d}$ when $\left(I_{s w i}\right)_{p}=$ $11.5 \mathrm{~mA}, L_{g}=1 \mathrm{nH}, C_{d}=10 \mathrm{pF}$, and $R_{g}=2.2 \Omega$

\section{B. Noise Sensitivity as a Function of Rise Time}

The normalized sensitivity of the ground noise as a function of the rise time is determined in this section to evaluate the efficacy of reducing the parasitic inductance and increasing the decoupling capacitance on reducing the ground noise. The normalized sensitivity of the ground noise to a parameter $p_{i}$ is determined by

$$
S_{p_{i}}^{\left(V_{g n d}\right)_{p p}}=\lim _{\Delta p_{i} \rightarrow 0} \frac{\frac{\Delta\left(V_{g n d}\right)_{p p}}{\left(V_{g n d}\right)_{p p}}}{\frac{\Delta p_{i}}{p_{i}}}=\frac{p_{i}}{\left(V_{g n d}\right)_{p p}} \frac{\partial\left(V_{g n d}\right)_{p p}}{\partial p_{i}} .
$$

The normalized sensitivity of the ground noise as a function of rise time, as determined by (20), is shown in Fig. 5. The sensitivity of the noise to the decoupling capacitance is high at small rise times and decreases with increasing rise time. Alternatively, the sensitivity of the noise to the parasitic inductance is low at small rise times and increases with longer rise times. Increasing the decoupling capacitance 
TABLE I

COMPARISON OF THE PEAK-TO-PEAK GROUND NOISE OBTAINED AT $t_{r}=2 \sqrt{\left(L_{g} C_{d}\right)}$ AND THE MAXIMUM NOISE FOR DIFFERENT PARASITIC GROUND NETWORK IMPEDANCES.

\begin{tabular}{c|c|c|c|c|c|c|c}
\hline $\begin{array}{c}\left(I_{s w i}\right)_{p} \\
\mathrm{~mA}\end{array}$ & $\begin{array}{c}L_{g} \\
(\mathrm{nH})\end{array}$ & $\begin{array}{c}C_{d} \\
(\mathrm{pF})\end{array}$ & $\begin{array}{c}R_{g} \\
(\mathrm{ohm})\end{array}$ & $\begin{array}{c}R_{d} \\
(\mathrm{ohm})\end{array}$ & $\begin{array}{c}\text { Ground noise at } \\
t_{r}=2 \sqrt{L_{g} C_{d}}(\mathrm{mV})\end{array}$ & $\begin{array}{c}\text { Maximum ground noise } \\
(\mathrm{mV})\end{array}$ & $\begin{array}{c}\text { Error } \\
(\%)\end{array}$ \\
\hline 11.5 & 0.25 & 10 & 2.2 & 0.1 & 44 & 46.2 & 4.8 \\
\hline 11.5 & 0.5 & 10 & 2.2 & 0.1 & 66.3 & 67.8 & 2.2 \\
\hline 11.5 & 1 & 10 & 2.2 & 0.1 & 98.5 & 99.8 & 1.3 \\
\hline 11.5 & 1 & 15 & 2.2 & 0.1 & 78.3 & 67.6 & 1.6 \\
\hline 11.5 & 1 & 20 & 2.2 & 0.1 & 66.3 & 64.8 & 2.2 \\
\hline 11.5 & 1 & 20 & 4 & 0.1 & 59.4 & 70.2 & 21.5 \\
\hline 11.5 & 1 & 20 & 6 & 0.1 & 55.1 & 68.6 & 21.3 \\
\hline 11.5 & 1 & 20 & 6 & 1 & 54 & 67.2 & 21.4 \\
\hline 11.5 & 1 & 20 & 6 & 2 & 52.8 & &
\end{tabular}

TABLE III

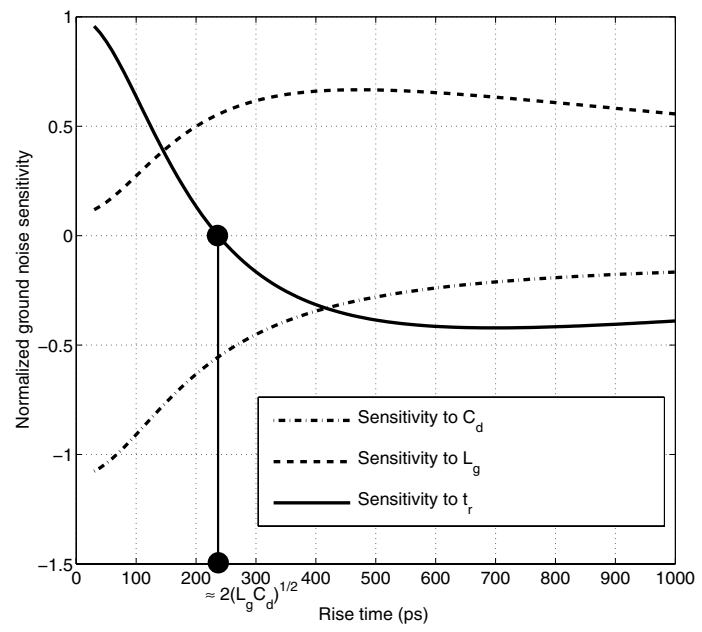

Fig. 5. Normalized sensitivity of the ground noise as a function of rise time when $\left(I_{s w i}\right)_{p}=11.5 \mathrm{~mA}, L_{g}=1 \mathrm{nH}, C_{d}=10 \mathrm{pF}, R_{g}=2.2 \Omega$, and $R_{d}=0.1$ $\Omega$

\section{TABLE II}

THE EFFECT OF THE DECOUPLING CAPACITANCE ON REDUCING THE PEAK-TO-PEAK GROUND NOISE. $L_{g}=1 n H$.

\begin{tabular}{c|c|c|c}
\hline Rise time (ps) & $C_{d}=10 p F$ & $C_{d}=20 p F$ & Reduction \\
\hline 70 & 57.8 & 28.2 & $51.2 \%$ \\
\hline $200=2 \sqrt{L_{g} C_{d}}$ & 98.7 & 59.5 & $39.7 \%$ \\
\hline 400 & 91.1 & 67.7 & $25.7 \%$ \\
\hline 800 & 69.4 & 58.7 & $15.4 \%$ \\
\hline
\end{tabular}

is therefore effective in reducing the noise for $t_{r} \leq 2 \sqrt{L_{g} C_{d}}$. Alternatively, reducing the parasitic inductance is effective for $t_{r} \geq 2 \sqrt{L_{g} C_{d}}$. This behavior is due to the changing ratio of the capacitive and inductive currents with respect to the rise time, as shown in Fig. 2. The effect of the decoupling capacitance and parasitic inductance on the ground noise is listed, respectively, in Tables II and III for different rise times. At $t_{r}=70 \mathrm{ps}$, doubling the decoupling capacitance reduces the noise by $51.2 \%$, and only $25.7 \%$ when $t_{r}=400 \mathrm{ps}$. Halving the parasitic inductance, however, reduces the noise by only $15.4 \%$ when $t_{r}=70 \mathrm{ps}$, and $35.6 \%$ when $t_{r}=400$ $p s$. Note that the sensitivity to the rise time crosses over at zero when $t_{r} \approx 2 \sqrt{\left(L_{g} C_{d}\right)}$, demonstrating the non-monotonic dependence, as described in Section III-A.
THE EFFECT OF THE PARASITIC INDUCTANCE ON REDUCING THE PEAK-TO-PEAK GROUND NOISE. $C_{d}=10 p F$.

\begin{tabular}{c|c|c|c}
\hline Rise time $(\mathrm{ps})$ & $L_{g}=1 \mathrm{nH}$ & $L_{g}=0.5 \mathrm{nH}$ & Reduction \\
\hline 70 & 57.8 & 48.9 & $15.4 \%$ \\
\hline $200=2 \sqrt{L_{g} C_{d}}$ & 98.7 & 67.7 & $31.4 \%$ \\
\hline 400 & 91.1 & 58.7 & $35.6 \%$ \\
\hline 800 & 69.4 & 48.2 & $30.5 \%$ \\
\hline
\end{tabular}

\section{CONCLUSIONS}

The non-monotonic dependence of the power/ground noise on the rise time is shown for an inductive power distribution network with a decoupling capacitance. The power/ground interconnect is modeled as a series $R L$ impedance. The decoupling capacitance is modeled as a capacitance in series with a resistance. The model captures the dependence of noise on the rise time with sufficient accuracy, as compared to SPICE simulations. The worst case rise time producing the maximum peak-to-peak noise is presented based on this model. The sensitivity of the noise on the decoupling capacitance and parasitic inductance is also investigated. A decoupling capacitance is shown to efficiently reduce the noise for $t_{r} \leq$ $2 \sqrt{L_{g} C_{d}}$. Alternatively, reducing the parasitic inductance is effective for $t_{r} \geq 2 \sqrt{L_{g} C_{d}}$.

\section{REFERENCES}

[1] M. Popovich, A. V. Mezhiba, and E. G. Friedman, Power Distribution Networks with On-Chip Decoupling Capacitors, Springer Verlag, 2008.

[2] P. Larsson, "di/dt Noise in CMOS Integrated Circuits," Analog Integrated Circuits and Signal Processing, Vol. 14, No. 2, pp. 113-129, September 1997.

[3] S. H. Hashemi, P. A. Sandborn, D. Disko, and R. Evans, "The Close Attached Capacitor: A Solution to Switching Noise Problems," IEEE Transactions on Components, Hybrids, and Manufacturing Technology, Vol. 15, No. 6, pp. 1056-1063, December 1992.

[4] L. D. Smith, R. E. Anderson, D. W. Forehand, T. J. Pelc, and T. Roy, "Power Distribution System Design Methodology and Capacitor Selection for Modern CMOS Technology," IEEE Transactions on Advanced Packaging, Vol. 22, No. 3, pp. 284-291, August 1999.

[5] M. Popovich and E. G. Friedman, "Decoupling Capacitors for MultiVoltage Power Distribution Systems," IEEE Transactions on Very Large Scale Integration (VLSI) Systems, Vol. 14, No. 3, pp. 217-228, March 2006.

[6] P. Larsson, "Resonance and Damping in CMOS Circuits with On-Chip Decoupling Capacitance," IEEE Transactions on Circuits and Systems I: Fundamental Theory and Applications, Vol. 45, No. 8, pp. 849-858, August 1998.

[7] H. H. Chen and J. S. Neely, "Interconnect and Circuit Modeling Techniques for Full-Chip Power Supply Noise Analysis," IEEE Transactions on Components, Packaging, and Manufacturing Technology, Vol. 21, No. 3, pp. 209-215, August 1998. 